\title{
Reflexiones en torno a la enseñanza de la bibliotecología
}

Ramiro Lafuente López*

Estela Morales Campos*

\begin{abstract}
RESUMEN
Se exponen algunas reflexiones sobre la enseñanza universitaria de la Bibliotecología en el nivel licenciatura, con relación a la conceptualización actual de la disciplina y el conocimiento teórico y práctico que se le debe ofrecer al estudiante para poderse desarrollar profesionalmente.
\end{abstract}

\begin{abstract}
Some thoughts are presented on the teaching of library science at the undergraduate level, and the way in which this field is perceived at present as web as the theoretical and practical knowledge which should be offered to students in order for them to be able to develop themselves professionally.
\end{abstract}

$\mathrm{E}_{\mathrm{c}}^{\mathrm{l}}$ plantearse los problemas relativos a la enseñanza de la Bibliotecología implica necesariamente buscar una correspondencia adecuada entre el qué enseñar, cómo enseñar y para qué enseñar, cuestiones que no son factibles de resolver fácilmente debido a que la enseñanza de una disciplina como la Bibliotecología involucra una diversidad muy amplia de aspectos. En este ensayo nos ocupamos de la enseñanza de la Bibliotecología subordinándola a la idea de que le enseñanza de una disciplina en el entorno universitario de una licenciatura, involucra primordialmente la necesidad de dotar a un individuo de ls conocimientos indispensables para que puede desempeñarse profesionalmente y hacer de este desempeño una actividad productiva tanto para él mismo como para el ámbito social donde se encuentre.

El determinar los objetivos a cumplir con la enseñanza de la Bibliotecología puede fectuarse desde una perspectiva en donde se considere que el conocimiento bibliotecológico que se debe enseñar a un individuo, debe buscar transmitir una visión integral de la Bibliotecología como disciplina, con la finalidad de que el alumno cuente con los elementos indispensables, para identificar aquel tipo de problemas factibles de ser resueltos con los conocimientos que tiene. Esta concepción, con- duce a centrar la enseñanza en aspectos formativos destinados a dotar a un individuo de la capacidad necesaria para:

a) identificar y comprender los fenómenos propios del ámbito bibliotecológico con objeto de que sepa como enfrentar un fenómeno.

b) Ser capaz de aplicar todos los conocimientos que tiene para la solución de casos específicos que se le presenten en el ejercicio profesional.

Para que un individuo sea capaz de identificar un fenómeno y aplicar los conocimientos que tiene en la solución de los casos específicos que le plantea el ejercicio profesional, requiere en primer término de una formación que lo dote de una visión integral acerca de lo que es la disciplina en sí y de todos los fenómenos que abarca.

La apreciación de los fenómenos relativos al ámbito bibliotecológico depende de la concepción que se tenga de la disciplina, si consideramos a la Bibliotecología como la ciencia que estudia: el registro y flujo del conocimiento y de la información; así como la circulación social de los medios que la contienen para hacer posible su uso y organización. ${ }^{1}$ Entonces el estudiante requeriría saber: Qué son y cómo se producen los registros del conocimiento y de la información (libros, folletos, revistas, videos y otros), cómo circulan y se usan; y cuáles son las formas que adopta el flujo de la información en una sociedad determinada. Debe además, aprender a seleccionar e interpretar documentos, para crear representaciones de los mismos. Es indispensable que tenga una idea muy clara de lo que significa clasificar en el ámbito bibliotecológico, con la finalidad de que pueda utilizar los sistemas clasificadores; y que entienda que como consecuencia de la formación de colecciones y un óptimo manejo de las representaciones de los documentos, se puede facilitar el flujo de información y el acceso a los documentos, así como prestar diversos tipos de servicios que deben responder a las cualificaciones que se hagan respecto del uso que una comunidad efectúe del conocimiento y la información contenida en los documentos.

El proporcionar una formación de este tipo requiere: agrupar los fenómenos en áreas de conocimiento que permitan aglutinar con propósitos didácticos fenómenos afines o que sean necesarios como antecedente conceptual para la comprensión o explicación de otros. La determinación de áreas de conocimiento en el caso de la Bibliotecología presenta una peculiaridad que es necesario atender con el debido detenimiento. Como los conocimientos de la disciplina han evo-

* Investigadores del Centro Universitario de Investigaciones Bibliotecológicas de la UNAM. 
lucionado rápidamente a partir de la década de los 80, no existe en estos momentos una clasificación de los conocimientos de la disciplina que esté completamente validada y sea de uso común. En la literatura existen diversas propuestas sobre estas cuestiones, así como trabajos acerca de lo que debe comprender la enseñanza de la disciplina y los programas de investigación, los cuales no hacen referencia al marco conceptual de la disciplina.

Sin embargo, la organización de los conocimientos bibliotecológicos que tiene unamayor aceptación aún corresponde a los esquemas clásicos de la organización técnica de la Biblioteca, a saber: Servicios, Catalogación, clasificación, y Usuarios. Situación que dificulta los planteamientos acerca de la enseñanza, puesto que es necesario atender a dos cuestiones: la clasificación, jerarquización e interrelación del conocimiento bibliotecológico, y la estructuración de áreas de enseñanza.

En la práctica se ha optado por seguir manejando el esquema clásico de jerarquización del conocimiento bibliotecológico y supeditado a la biblioteca. Sin embargo, como se presenta el problema de actualizar la enseñanza e incorporar los nuevos conocimientos, entonces se ha recurrido a insertar en el esquema clásico los nuevos conocimientos, que si bien resulta una solución pragmática e inmediatista, no es la más adecuada a los propósitos que se persiguen de efectivamente actualizar la enseñanza para hacerla acorde con el avance de los fenómenos sociales y tecnológicos y los objetivos de los estudios de licenciatura.

La validación de la jerarquización e interrelación del conocimiento bibliotecológico es un aspecto fundamental para lograr una adecuada construcción de las áreas de enseñanza,puesto que la que permite obtener las pautas para que la definición de áreas de enseñanza efectivamente posibilite el transmitir al alumno una visión integradora del conocimiento bibliotecológico. Existen otro tipo de problemas relativos al orden en que las asignaturas deberán agruparlos fenómenos. Una alternativa de solución a este problema, se encuentra en dirección de establecer prioridades lógicas de enseñanza, basadas en la idea de identificar conceptos primarios y aumentar gradualmente la complejidad del manejo conceptual, de tal manera que el alumno en los últimos años sea capaz de manejar e interrelacionar los conceptos relativos a la explicación o comprensión de los fenómenos motivo de la enseñanza.

El contenido de algunas de las asignaturas atendería desde luego a la enseñanza de lo que son los fenómenos, los principios, las teorías, con la intención de que el alumno aprenda el desarrollo de la disciplina, cuáles son sus supuestos y fundamentos y qué valor tiene el conocimiento bibliotecológico. Es necesario también, enseñar que los fenómenos bibliotecológicos se presentan en un entorno social determinado y que durante el transcurso de su evolución han generado instituciones relevantes para la sociedad que aplican el conocimiento bibliotecológico y representan un aspecto fundamental para la preservación y difusión de la cultura como son las Bibliotecas Públicas, las Bibliotecas Nacionales, las Bibliotecas Universitarias y las Bibliotecas Especializadas; y esta misma sociedad en su desarrollo y adopción de nuevas formas de enfrentar fenómenos presenta sus instituciones natas y crea nuevas unidades de información y todas en su conjunto incorporan las novedades tecnológicas y científicas.

La aproximación al entorno social de los fenómenos bibliotecológicos es factible realizarla ubicando en una primera instancia cuáles son los fenómenos sociales que generan la creación, reproducción y difusión de conocimientos e información por medio de documentos, fenómenos primarios $\sin$ los cuales no puede presentarse el fenómeno bibliotecológico, el cual arranca precisamente de la existencia misma de conocimientos e información registrados por algún medio. La creación, el flujo, reproducción y difusión de registros del conocimiento y la información, podemos englobarlos como un fenómeno al que podemos denominar como el ciclo de circulación social de documentos. Es necesario transmitir a los alumnos que su actuación profesional requiere contar con una percepción adecuada acerca de la forma en cómo puede ubicar su actividad como parte del flujo de información y ciclo de circulación social de documentos en el entorno de la sociedad en la que pretenda ejercerla.
Al proporcionar al alumno una concepción integral de los fenómenos que comprende la Bibliotecología se pretende darle una formación a partir de la cual pueda diseñar, desarrollar y operar unidades y servicios de información adecuados a los requerimientos de la sociedad en donde se pretende se desenvuelva su vida profesional; se busca una formación flexible que evite ligarlo al uso particular de una tecnología o a los saberes requeridos por un segmento del mercado de trabajo.

Una adecuada formación del bibliotecólogo requiere de un nivel autónomo constituido por elementos cognoscitivos que son esencialmente abstractos y que dan lugar a una actividad teórica, pero que para lograr una sana actividad profesional el conocimiento teórico debe equilibrarse con el saber hacer. Sin embargo el saber hacer, no debe de enseñarse como un conjunto de procedimientos repetitivos y mecánicos, desprovistos de toda cualificación, sino como un hacer del que conocemos el cómo y el para qué hacerlos, lo cual implica necesaria y esencialmente un dominio del conocimiento de los fenómenos bibliotecológicos.

Se persigue que la enseñanza de los principios y teorías esté orientada a la comprensión de la naturaleza de los fenómenos, con la intención de que el alumno se apropie de los conocimientos suficientes para identificar el fenómeno, pero atendiendo en todo momento a que sea capaz de vincular estos conocimientos con su aplicabilidad pragmática. No se trata, por ejemplo, de que aprenda los fundamentos de la clasificación, sino que los aprenda con la intención de comprender qué son y para qué sirven los sistemas clasificadores más importantes con objeto de que tenga las habilidades suficientes para aplicarlos a la solución de casos específicos que habrá de encontrar en su vida profesional.

La enseñanza de los principios y fundamentos pueden realizarse a través de asignaturas y seminarios, en los cuales deberá enseñarse al alumno no sólo a identificar el fenómeno bibliotecológico sino también a valerse de aquellos conocimientos que se generan en otras disciplinas para comprender y explicar los fenómenos bibliotecológicos. Sería el caso por ejemplo, de la enseñanza de las teorías de la comunicación, que debiera efectuarse desde 
la perspectiva de mostrar al alumno la utilidad para implementar y manejar servicios de información para comprender diversos problemas relativos al usuario. Podría ser también el caso de la enseñanza de la linguiística o la lógica, que deben estar orientadas a ayudar a la comprensión y explicación de fenómenos inherentes a la clasificación en el ámbito bibliotecológico. Sin embargo, la aplicabilidad pragmática de estos conocimientos requiere de un área de laboratorios donde se conduzca al alumno hacia el desarrollo de habilidades para la aplicación de los conocimientos que está adquiriendo en las asignaturas y seminarios.

La enseñanza de la aplicabilidad pragmática del conocimiento bibliotecológico por medio de laboratorios requiere que por sí mismos, tengan objetivos didácticos encaminados a buscar la integración de conocimientos para la solución de problemas específicos, de tal manera que el laboratorio obligue al alumno no sólo a operar las distintas tecnologías sino a valerse de los principios y fundamentos adquiridos respecto de un fenómeno, en la solución de problemas específicos.

La configuración de laboratorios tendría que responder a la idea de que el alumno sea capaz de diseñar servicios, organizar unidades e información, construir catálogos manuales o automatizados, u organizar colecciones. Evitar la idea de que el laboratorio sirva únicamente para que el alumno efectúe "prácticas", su finalidad didáctica debe ser el plantearse problemas y su posible solución valiéndose de las herramientas que se le proporcionen en el laboratorio. De esta manera, su finalidad sería permitir al alumno integrar su conocimiento por medio de la realización de un proyecto determinado, por ejemplo, un laboratorio de catalogación automatizada no sólo debería tener como finalidad el aprender a operar y practicar con determinado tipo de paquetes de automatización. El laboratorio debe permitir que el alumno se plantee un servicio de información y lo resuelva por medio del uso de un determinado tipo de paquetes de aplicación y de una adecuada aplicación de las reglas y esquemas de clasificación.

Parte de esta enseñanza son los seminarios dentro de los cuales sería conveniente contar con alguno de ellos para iniciar al estudiante a un trabajo cercano a la inves- tigación. Los seminarios deberían tener valor curricular pero no deben de tener ningún tema asignado, el tema sería conveniente que lo asignaran cada semestre los profesores e investigadores de carrera, y deberá estar vinculado a sus trabajos de investigación. El seminario debería tener como objetivo el abrir un espacio para que el alumno enfrente a otro tipo de abordaje de los problemas que le sirvan de base para aprender a manejar conocimientos con un alto grado de abstracción.

El contemplar la enseñanza de la bibliotecología en el sentido antes mencionado, implica buscar la formación de un alumno a partir de aglutinar los conocimientos disponibles con la finalidad de darle una formación que le permita ejercer su profesión en un mercado de trabajo sujeto a constantes cambios. $\mathrm{Al}$ abordar el problema de la formación de áreas de enseñanza de la Bibliotecología, optamos por agrupar fenómenos semejantes o interrelacionados, con la idea de que la enseñanza se realice conforme a un orden de prioridades lógicas, a fon de que al alumno se le proporcionen los conocimientos que requiere para comprender un fenómeno, así como plantearse un problema y la forma de solucionarlo. De esta manera, el alumno aprenderá los elementos suficientes para comprender y explicar las relaciones que existen entre todos los fenómenos que se le enseñen.

La enseñanza se aprecia a partir de la definición de áreas en donde se agrupan fenómenos con la intención de cumplir las finalidades que se indican en cada una de ellas. Las áreas que son parte distintiva de la disciplina y se considerarán como objeto de enseñanza en un nivel universitario de licenciatura son las siguientes:

ÁREA 1: CONOCIMIENTO E INFORMACIÓN

ÁREA 2: USO DEL CONOCIMIENTO Y DE LA INFORMACIÓN.

ÁREA 3: SERVICIOS DE INFORMACIÓN.

ÁREA 4: INDUSTRIA EDITORIAL Y DE LA INFORMACIÓN.

ÁREA 5: TECNOLOGÍA.

ÁREA 6: ORGANIZACIÓN DE BIBLIOTECAS Y UNIDADES DE INFORMACIÓN.

ÁREA 7: SISTEMAS DE CLASIFICACIÓN BIBLIOTECOLÓGICOS.

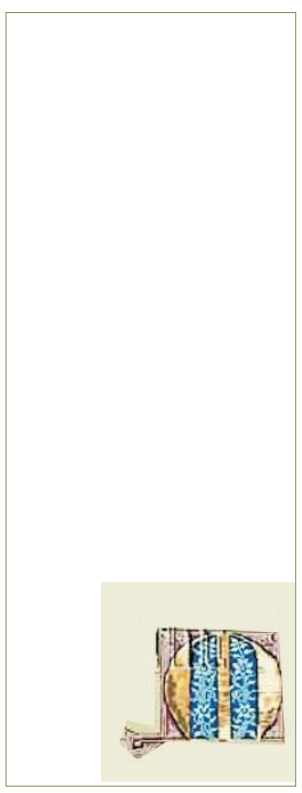

\section{Abordamos el problema de la formación de áreas de enseñanza de la}

Bibliotecología... con la idea de que la enseñanza se realice conforme a un orden de prioridades lógicas, a fin de que al alumno se le proporcionen los conocimientos que requiere para comprender un fenómeno, así como plantearse un problema y la forma de solucionarlo. 
ÁREA 8: METODOLOGÍA DE LA INVESTIGACIÓN BIBLIOTECOLÓGICA.

Las finalidades a cumplir en cada una de las áreas, se detallan a continuación:

\section{ÁREA 1 \\ CONOCIMIENTO E INFORMACIÓN}

\section{FINALIDADES:}

Conceptuar el conocimiento y la información como fenómenos que competen el estudio de varias disciplinas. Distinguir que el registro del conocimiento y de la información están en relación a su flujo, uso y organización, y son aspectos inherentes al ámbito bibliotecológico. Para dar cumplimiento a este objetivo es necesario enfatizar las diferencias que existen entre conocimiento e información, y las diversas maneras que existen para su registro. ón, y las diversas maneras que existen para su registro.

Para dar cumplimiento a este objetivo la currícula deberá de contemplar:

a) El estudio de qué se entiende por conocimiento y qué por información,

b) Las diversas formas y medios de comunicación que se utilizan para publicar y dar a conocer el conocimiento y la información,

c) El análisis de cómo el registro y organización del conocimiento y de la información adopta diversas modalidades de uso en distintas épocas y lugares.

d) El estudio del conocimiento y de la información desde diversas disciplinas y en especial como lo aborda la bibliotecología.

e) El estudio de las formas que adopta el flujo de información en distintas épocas y lugares.

\section{ÁREA 2 \\ USO DEL CONOCIMIENTO DE LA INFORMACIÓN}

\section{FINALIDADES}

Conceptualizar cómo el uso y organización de los registros del conocimiento y de la información es un fenómeno en donde intervienen factores de diversa índole, a saber: actitudes personales y de grupo, procesos de adquisición del conocimiento relacionados con el hábito de lectura y formas de manejo de información, procesos de comunicación determinación de necesidades de conocimiento e información, procesos de enseñanza aprendizaje, y otros, que facilitan o dificultan la implementación de servicios de información.

Para dar cumplimiento a este objetivo la currícula deberá de contemplar:

a) Los métodos para interpretar los diversos significados del discurso con objeto de que el alumno sea capaz de identificar los problemas que se presentan en la descripción, clasificación, elaboración de resúmenes, servicios bibliográficos, de difusión y otros.

b) La utilización de los diferentes modelos de comunicación, como un medio para lograr la difusión del conocimiento y de la información. Enfatizar cómo el uso de determinados modelos e comunicación facilitan o dificultan el acceso al conocimiento y la información.

c) Las diferentes tipologías de usuario y su relación con el entorno social, el prestador de servicios, la colección, la administración de los servicios y las finalidades de la institución que presta los servicios.

d) Los procesos de enseñanza aprendizaje relativos al uso del conocimiento e información, como pueden ser la lectura, el uso de información, y la formación de usuarios.

e) El valor de la lectura y del libro como instrumentos para el registro y difusión del conocimiento y la información.

\section{ÁREA 3 \\ SERVICIOS Y DE INFORMACIÓN}

\section{FINALIDADES:}

Conceptualizar las diversas formas y modalidades de los servicios de información, tanto actuales como aquellos que sea previsible se implemente o estén por implementarse en el futuro. Enfatizar las diferencias y semejanzas entre los servicios que prestan instituciones sociales con finalidades que atienden al interés público como las Bibliotecas y los servicios que es factible implementar conforme a necesidades específicas en cuanto al uso del conocimiento y la información dependiendo de circunstancias temporales, contextos sociales, cumplimiento de fines institucionales y otros.

Para dar cumplimiento a este objetivo la currícula deberá de contemplar:

a) la conceptualización del servicio de información.

b) Las diferentes modalidades que adoptan los servicios de información. Relacionar las diferentes modalidades que pueden adoptar los servicios de información con el uso de los diferentes sistemas de clasificación documental y con las formas que adopta la organización administrativa de los servicios.

c) Los servicios de información y su implementación a través de instituciones de servicio público o de carácter privado. Su creación como fenómenos aislados, o como parte de una organización.

d) Costo económico y recursos materiales y humanos requeridos para la prestación y organización de estos servicios.

e) El carácter de preservación y difusión de la cultura que representan los servicios que prestan las Bibliotecas Públicas, las Bibliotecas Nacionales, las Bibliotecas Universitarias, y las Bibliotecas Especializadas. Enfatizando la historicidad de este tipo de instituciones y los fenómenos sociales vinculados a las mismas.

f) La evolución y formas de prestación de servicios de información privados y comerciales.

g) Los servicios de información y la formación de colecciones documentales acorde a la naturaleza de los servicios de información que se prestan. Relaciones entre el tipo de documento y factores como: el tema, nivel de actualización de información que se requiera, las formas de publicación, y otros, para la conformación de acervos.

h) Los servicios de información como parte de un contexto cultural y los problemas relativos al derecho de información, acumulación y difusión social del conocimiento: alteraciones que producen en tradiciones de comunicación y acumulación del conocimiento en una comunidad, problemas 
de carácter ético en la prestación de los servicios.

\section{ÁREA 4 \\ INDUSTRIA EDITORIAL Y DE LA INFORMACIÓN}

\section{FINALIDADES:}

Conceptualizar el ciclo de circulación social de conocimientos e información, por medio de la creación, producción, difusión de documentos, ubicando el desarrollo de colecciones como parte de los fenómenos y actividades sociales inherentes al ciclo de circulación documental.

Para dar cumplimiento a este objetivo la currícula deberá de contemplar:

a) conceptualización de la naturaleza de la industria de la información y de la industria editorial, el editor, el librero, el lector, el mercado del libro, el mercado de la información.

b) Análisis de los fenómenos económicos que permiten comprender el mercado de la información: valor de la información como mercancía, análisis financiero de los servicios de información, mercadotecnia de los servicios de información.

c) Análisis de los factores económicos que intervienen en la industria editorial: las condiciones sociales y económicas que propician el desarrollo de la industria editorial, y de la información y de la influencia que tienen estas industrias en la conformación de los servicios de información.

d) Los componentes de los costos en cuanto a la presentación de servicios de información enfatizando el análisis financiero de los costos que representa el mantener la infraestructura administrativa para la prestación de servicios de información.

e) Técnicas presupuestales para el financiamiento y control contable para la implementación, mantenimiento y desarrollo de los servicios de información, a partir del establecimiento de relaciones entre los costos de los servicios y los costos de los insumos que proporciona la industria editorial y de la información.

f) Principios y estrategias racionales para la búsqueda, evaluación y discriminación de fuentes de información para el desarrollo de colecciones. Así como las relaciones entre las fuentes de información, el desarrollo de colecciones y las formas de satisfacer demandas de información.

\section{ÁREA 5 TECNOLOGÍA}

\section{FINALIDADES:}

Analizar las diferentes tecnologías diseñadas específicamente para la organización de documentos y de las representaciones que se realicen de las mismas. Así como la tecnología de automatización como auxiliar en la implementación y operación de servicios de información.

Para dar cumplimiento a este objetivo la currícula deberá de contemplar:

A) Fundamentos y operación de aquellas tecnologías que sean útiles para el desempeño profesional, en relación a:

a1) El tipo de mobiliario para acomodar y guardar documentos, y la relación que guarda con la forma de los documentos, su clasificación, los procedimientos de control administrativo y los servicios de préstamo de documentos.

a2) La conservación y restauración de materiales documentales.

a3) Aprovechamiento y organización del espacio físico para el acomodo de colecciones y la prestación de servicios de información.

a4) Reproducción de documentos.

a5) Los procesos físicos del libro y otros documentos como instrumentos para el control administrativo de las colecciones, enfatizando las relaciones que existen entre los procesos físicos y diversos procedimientos de control administrativo de los acervos.

B) Aplicación de la automatización a la organización y difusión de los servicios de información.

b1) La automatización como instrumento de control administrativo de los procedimientos para operar servicios de información.

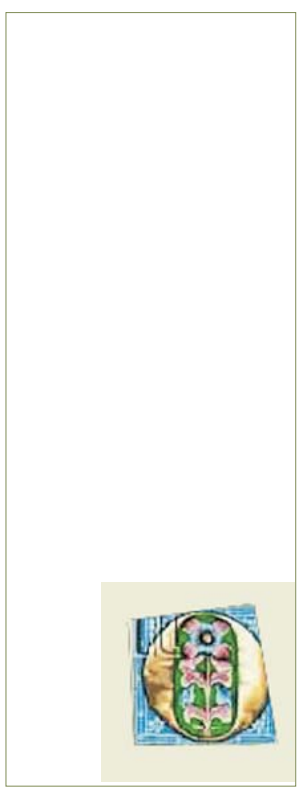

Distinguir que el registro del conocimiento y de la información están en relación a su flujo, uso y organización, y son aspectos inherentes al ámbito bibliotecológico. 
b2) Operación de los sistemas de automatización de servicios de información disponibles.

b3) Principios científicos y tecnológicos de los sistemas automatizados para el manejo de datos bibliográficos.

b4) Telecomunicaciones y redes de información.

b5) Formatos internacionales para el almacenamiento de información bibiográfica. Automatización de la catalgación.

\section{ÁREA 6 \\ ORGANIZACIÓN DE \\ BIBLIOTECAS Y UNIDADES DE INFORMACIÓN}

\section{FINALIDADES:}

Los servicios de información requieren de una organización administrativa eficiente y diseñada específicamente para cumplir determinadas metas y fines.

Que la organización de bibliotecas y unidades de información puede adoptar diversas modalidades que pueden ser transitorias o institucionales.

Para dar cumplimiento a este objetivo la cirrícula deberá de contemplar:

a) las diferentes técnicas de organización y administración tradicionales y flexibles, útiles a las bibliotecas y otras unidades de información, los servicios de información y los sistemas de información.

b) las diferentes modalidades organizacionales que pueden adoptar los servicios de información, de acuerdo al tipo de documento, al usuario, a las condiciones sociales y/o las finalidades institucionales (v.g. centro de documentación, centro de información, biblioteca, y otros).

c) Las relaciones humanas y laborales que inciden en la organización, oferta y calidad de los servicios de información.

d) La relación que existe entre los sistemas de organización administrativa y los sistemas de clasificación de acervos y prestación de servicios de información.

e) Análisis de los ciclos de control administrativo de:
- la adquisición de documentos hasta su integración a los acervos

- los servicios de préstamo de documento.

- las formas de comunicación con el usuario.

- la organización y procedimiento de control de redes y servicios de información en línea.

- los sistemas de inventarios.

f) Técnicas presupuestales, de análisis financiero, y de control contable, par ala implementación, mantenimiento y desarrollo de los servicios de información. Establecer las relaciones que existen entre los costos de los servicios, los costos de los insumos que proporciona la industria editorial y de la información, y el costo de los recursos humanos y materiales necesarios para la creación y operación de los servicios de información.

\section{ÁREA 7 \\ SISTEMAS DE CLASIFICACIÓN BIBLIOTECOLÓGICOS}

\section{FINALIDADES:}

La naturaleza lógica de los sistemas de clasificación bibliotecológica con objeto de enfatizar el uso que se hace de las relaciones formales, la significación, y los procesos de inferencia en la construcción y manejo de los sistemas bibliotecológicos de clasificación.

Principios básicos de la clasificación de los contenidos de los documentos como elemento indispensable para la organización de colecciones y la prestación de servicios de información.

Para dar cumplimiento a este objetivo la currícula deberá de contemplar:

a) las teorías de la clasificación vinculados a la organización documental.

b) Los sistemas para clasificar los diferentes medios en que se presenta el conocimiento y la información.

c) La naturaleza de los sistemas bibliotecológicos en cuanto a las diversas modalidades que presentan y señalar qué utilidad tiene cada uno de ellos para organizar acervos, catálogos, servicios bibliográficos, diseminación selectiva, y otros. d) La relación entre la clasificación y los servicios de información. La organización física de los acervos y su estrecha relación con los servicios de información, los sistemas de clasificación, el tipo de colecciones.

e) Las diversas formas en que se pueden aplicar los sistemas de clasificación para resolver problemas particulares de clasificación de documentos, para atender al cumplimiento de finalidades específicas.

f) Organización de colecciones, catálogos, índices y cualquier herramienta útil en la recuperación de información.

g) La formación de catálogos tanto manuales como automatizados como un medio para organizar las representaciones de los contenidos de los documentos.

h) Los lenguajes documentales

i) El análisis de contenidos documentales para clasificar acervos y prestar servicios de información

j) La elaboración de resúmenes en el ámbito bibliotecológico a partir de la identificación e interpretación de los elementos significativos del discurso para representar por medio de un resumen los contenidos de los documentos.

k) Métodos para la compilación bibliográfica, incluyendo la elaboración de catálogos (de archivo, de editoriales, de librerías), las bibliografías (recomendadas, anotadas, comentadas y de resúmenes) y los índices de publicaciones de todo tipo de documentos manuscritos, impresos, microformas o soportes electrónicos.

\section{ÁREA 8 \\ METODOLOGÍA DE LA \\ INVESTIGACIÓN BIBLIOTECOLÓGICA}

\section{FINALIDADES:}

Conceptualizar el desarrollo del conocimiento Bibliotecológico, rescatando la historicidad tanto de los conceptos y técnicas como de la evolución misma de los fenómenos inherentes al ámbito bibliotecológico, enfatizando cómo es que se han 
creado diversos métodos para aproximarse al conocimiento de los fenómenos bibliotecológicos.

Para dar cumplimiento a este objetivo la currícula deberá de contemplar:

A) Las relaciones que existen entre la Bibliotecología y otras ciencias afines, y la ubicación que guarda la Bibliotecología en el entorno del quehacer académico y de investigación, enfatizando:

a1) La evolución del conocimiento bibliotecológico incluyendo las dife- rentes concepciones historiográficas que existen sobre la disciplina.

a2) El desarrollo de las concepciones acerca de la profesionalización del quehacer bibliotecario y de los servicios de información. Destacando la influencia que han tenido en la formación de estas concepciones tanto organismos de distinta nacionalidad como de caráceter internacional.

B) Métodos para la implementación y operación de servicios de informa- ción, y para la investigación de los fenómenos que le competen a la Bibliotecología, enfatizando:

b1) métodos de investigación propios al ámbito bibliotecológico.

b2) técnicas y métodos útiles al diseño y evaluación de servicios de información.

b3) métodos de enseñanza útiles a la promoción del uso de la información, hábitos de lectura y formación de usuarios de servicios de información.

\section{NOTAS}

1 Esta definición está basada en la que se elaboró en el Seminario de Currícula organizado por el CUIB en 1992 y se encuentra modificada y ampliada por los autores. Versión del Seminario: La Bibliotecología es la ciencia que estudia el registro del conocimiento y de la información con relación a su uso y organización.

\section{UNA NOTA BIBLIOGRÁFICA ...}

Estas reflexiones sobre a enseñanza de la Bibliotecología, además de ser una inquietud personal están apoyadas en una revisión exhaustiva de la literatura correspondiente tanto bibliotecológica como pedagógica, sin embargo creemos que para aquellos interesados en las tendencias respecto a la concepción acerca de la enseñanza de la Bibliotecología pueden ser útiles las siguientes referencias:

BORKO, HAROLD. “Comment”. En The Library Quarterly, vol. 2, no. 4 (Oct. 1964). P. 374-386.

CORNOVSKY, LEON, "Role of the Public Library: Implications for Library Education”, en: The Library Quaterly, vol. 24, no. 4 (Oct. 1964), pp. 315-325.

ENNIS PHILIP H. “The Study of the Use and Users of Recorded Knowledge”, en: The Library Quarterly, vol., 24, no. 4 (oct. 1964), pp. 305-314.

FERNÁNDEZ DE ZAMORA, ROSA MARÍA. "El Oficio de Bibliotecólogo", en Investigación Bibliotecológica, vol. 5, no. 10, enero-junio 1991, pp. 42-44.

FLOOD, MERRILL M. "The SystemsAproach to Library planning”, en: The Library Quarterly, vol. 24, no. 4, (Oct. 1964), pp. 326-338.

FORGIONNE, GUISSEPPI. "The college of information science: a mechanism to consolidate information science education", en: Education for Information 9(4) Dic. 1991, pp. 285-304.

FOSKETT, D. F. "Library education: the role of classification, indexing, and subject analysis", en: The Library Quarterly, vol. 24, no. 4, (Oct. 1964), pp. 362-373. 
LAU, JESUS. "La educación británica en el campo de la bibliotecología y ciencias de la información”, en: Investigación bibliotecológica, vol. 3, no. 6, enero-junio 1989, pp. 9-30.

HU, JAMES S. C. Library and information science education: An International Symposium, Klondon: the Scarecraw Press, 1987, 258 p.

MORALES CAMPOS, ESTELA, “La investigación bibliotecológica y su relación con el sector productivo”, en. Investigación bibliotecológica, vol. 4 no. 9, julio-dic., 1990, pp. 6-10.

PERALES OJEDA, ALICIA. "El ocaso del modelo norteamericano para la enseñanza/investigación Bibliotecológica y de la Ciencia de la Información”, en: IX Encuentro de Bibliotecología (9: 1992: Abril 27-30: México, D. F.), México, D. F., UNAM, Facultad de Filosofía y Letras, Colegio de Bibliotecología, 1992, 14 h.

SANDER, SUSANA. "Elementos histórico-teóricos para la indagación de la estructura teórica de la bibliotecología", en: Investigación bibliotecológica, vol. 3, no. 6, enero-junio 1989, pp. 31-37.

SETIEN, EMILIO. "Fundamentos teóricos y metodológicos de las investigaciones sobre la actividad de las bibliotecas públicas cubanas, Estado Actual y Perspectivas de desarrollo de esas investigaciones", en: Investigación bibliotecológica, vol. 3, no. 7, julio-dic., 1989, pp. 3-20.

SHERA, JESÉ H. Los fundamentos de a Educación Bibliotecológica., México: UNAM, Centro Universitario de Investigaciones Bibliotecológicas, 1990, 520 p.

SLAMECKA, VLADIMIR y MARTIMER TAUBE. "Theoretical Principles of Information Organization in Librarianship”, en: The Library Quaterly, vol. 24, no. 4 (Oct. 1964), pp. 352-361.

SWANSON, DON R. "The Intellectual Foundations of Library Education: Introduction”, en: The Library Quaterly, vol. 24, no. 4 (Oct. 1964), pp. 289-294.

WILSON, J. R., "The Development of Research in relation to library Schools, wn: Library Journal, vol. 58.

WILLIAMS, GORDON: "The Librarians Role in Development of Library Book Collections", en: The Library Quaterly, vol. 24, no. 4 (Oct, 1964).

En las referencias bibliográficas antes anotadas se recogen obras de los años 60, no es casual el que esto sea de esta manera, el esquema clásico de la Bibliotecología fue rebasado por la presencia de fenómenos que aparecieron como resultado de dar a la información un nuevo sentido y valor social, sobre todo a partir de la década de los 60. Algunos autores de esa época se percataron del fenómeno y presentan reflexiones que mueven a replantear el ámbito bibliotecológico. Representan un buen punto de partida para el análisis de estas cuestiones, sobre todo porque no tenían la fuerte y asfixiante influencia de las tendencias tecnologizantes que se van a presentar en la década de los ochenta, en donde pareciera ser que las formas de uso de la tecnología son el principio y el fin de todo fenómeno.

Respecto a la literatura mexicana sobre estas cuestiones es de suma utilidad la Bibliografía comentada sobre educación bibliotecológica en México, y cuya referencia es la siguiente:

RODRÍGUEZ GALLARDO, ADOLFO, et. al Bibliografía Comentada sobre Educación Bibliotecológica en México: México: CUIB-UNAM, 1987, 149 p.

Un somero análisis de la literatura mexicana sobre la enseñanza de la bibliotecología permite percibir un denominador común: la idea de que algo no camina bien en la enseñanza y capacitación que imparten las escuelas mexicanas de bibliotecología. También es factible inferir que se trata de señalar la presencia de nuevos fenómenos que están influyendo decisivamente en la transformación de los servicios bibliotecarios, sin embargo, se plantea explicarlos y resolverlos a partir del paradigma clásico de la Bibliotecología, que tiene a la biblioteca como objeto de estudio.

La insistencia en tratar de explicar los fenómenos del ámbito bibliotecológico a partir de un paradigma cuyos supuestos están siendo rebazados constantemente por la transformación de los servicios bibliotecarios, provocó el que los planteamientos derivaran en un deber ser que trata de que la realidad se ajuste a una serie de "normas" y "perfiles". Esta idea de replantear la enseñanza de la Bibliotecología a partir de un "perfil ideal" construido teniendo en mente el paradigma clásico de la Bibliotecología es una constante que se repite en la literatura mexicana sobre el tema. 
La bibliografía de Rodríguez Gallardo y compañía se publicó en 1987, a continuación se enlistan algunos artículos que pueden ser de interés, y que fueron publicados en fecha posterior a 1987:

ACEVEDO ROSAS, FELICIANO. Análisis comparativo de planes de estudio de la especialidad de biblioteconomía en México en el nivel de licenciatura. México: El Autor, 1983. 107 H. TESIS (Maestría en Biblioteconomía) ENBA.

CARRIÓN, GUADALUPE . Formación de profesionales de información a nivel de Maestría para los sectores productivo y de servicios. Venezuela: [S.E.], 1990. $22 \mathrm{H}$.

COLEGIO NACIONAL DE BIBLIOTECARIOS. Mesa Redonda sobre Formación de Recursos Humanos (5: 1990: Monterrey, N. L.), Monterrey, N. L.: Colegio Nacional de Bibliotecarios: Centro de Información Científica y Humanística, 1992.

COLEGIO NACIONAL DE BIBLIOTECARIOS. VI Mesa Redonda Sobre Formación de Recursos Humanos: Los Estudios Formales y otras Alternativas. (Cuernavaca, Morelos, 1-3 de Julio 1992), en prensa.

ESCUELA NACIONAL DE BIBLIOTECONOMÍA Y ARCHIVONOMÍA. Proyecto Académico; versión preliminar. México: 1991, 26 p.

GÓMEZ PÉREZ, GRISELDA y MA. DEL CARMEN MUÑOZ DE LA PEÑA. Los Egresados de Biblioteconomía. México [S.E.] 1988, 38 [13]H.: tbs. A la cabeza aparece Universidad de San Luis Potosí. División de Planeación.

IBARRA FERNÁNDEZ, ABRAHAM. "Reflexiones sobre el perfil del bibliotecólogo", en: Conferencia Binacional de Bibliotecas de las Californias (2: 1985: San diego, Calif.), Ponencias, San diego, Calif.: [S.E.], 1985, 11 p.

MORALES CAMPOS, ESTELA . "Bibliotecología e Información”, en: Boletín de la Asociación Andaluza de Bibliotecarios, Año 5, No. 5, jun. 1989, pp. 13-21.

MORALES CAMPOS, ESTELA. Testimonios de la Bibliotecología Mexicana: Educación 1915-1954, México: CUIB/UNAM, 1988, 110 p. (Monografías: 6).

OROZCO AGUIRRE, AURELIA. “Aspectos Curriculares”, en: Jornadas Mexicanas de Biblioteconomía (18: 1987: México, D.f.), Hacia el Siglo XXI: Memorias, México: AMBAC, 1989, pp. 343-348.

- - D DANIEL DE LIRA LUNA. Programa Trabajo de la Coordinación del Colegio de Bibliotecología, 1986-1987, México: Biblioteca Universitaria: Boletín Informativo de la Dirección General de Bibliotecas UNAM 1(4): 10-14, Oct./dic, 1986, 5 p.

OSORIO MORALES, JESÚS. Los antecedentes Trascendentales del Proceso Educativo Bibliotecológico en la UANL: 1966-1980, México: ITEMES: Revista de Información e Investigación Bibliotecológica 1(3): 9-14, Ene-abril, 1989.

RODRÍGUEZ GALLARDO, ADOLFO. "El Milagro Mexicano en Bibliotecología o la Ronda de las Generaciones", en: Seminario Nacional de bibliotecarios de México (1989: México, D.F.), Bibliotecología, Información y sociedad en México: Memorias, México: colegio Nacional de Bibliotecarios; CONACYT, 1989, H. 50-53.

SECRETARÍA DE EDUCACIÓN PÚBLICA y UNIVERSIDAD AUTÓNOMA DEL ESTADO DE MÉXICO. Reunión Nacional de Responsables de Bibliotecas de Universidades Públicas Estatales (3: 1986: Cuernavaca, Mor.), México: [S.E.], 1986, 39 H.

VERDUGO SÁNCHEZ, JOSÉ ALFREDO. La formación del bibliotecario mexicano a través del Bachillerato y la opción Técnica en Biblioteconomía. México: Investigación Bibliotecológica: archivonomía, bibliotecología e información 4(8): 13-19, ene/jun., 1990.

VILLAR BARRANCO, JOSÉ LUIS. "La formación de Profesionales de la Información dispuestos a Servirse de la Computadora", en: Seminario de ABIESI la Interacción de la Biblioteca y la Información (1985: san Luis Potosí, S. L. P.), México: ABIESI: UASLP: CONACYT, 1986, pp. 13-36. 